\title{
Análisis de las prácticas de entrevistas forenses durante la etapa de investigación por denuncias de delitos sexuales en contra de niños, niñas y adolescentes*
}

\section{Analysis of the practices in forensic interviews during the investigation phase of allegations of sexual offences against children and adolescents}

Recibido: 26 de abril de 2016/Aceptado:29 de septiembre de 2016

http://doi.org/10.17081/psico.20.37.2422

Palabras clave:

Entrevistas forenses, Delitos sexuales, Testimonio infantil.
Key words:

Forensic interviews,

Sexual offences, Child testimony.

\section{Resumen}

En este estudio se realizó un análisis de contenido tipo categoria 1 a 81 entrevistas realizadas por la Policía Judicial durante la etapa de investigación en denuncias por delitos sexuales, siendo las presuntas víctimas niños, niñas y adolescentes. Se analizó el uso de protocolos, la profesión de las personas encargadas de realizar las entrevistas, los estilos para iniciar las entrevistas, las estrategias para construir rapport, las estrategias para evaluar las competencias de los entrevistados como testigos y las estrategias para evaluar la sugestionabilidad. Se observa que en las prácticas de las entrevistas no se cumplen los procedimientos sugeridos en las distintas guías y protocolos y, por tanto, se comenten los errores que se intentan subsanar con el uso de dichas guías y protocolos.

\begin{abstract}
In this study, a categorical content analysis was performed to 81 interviews conducted by the Judicial Police during the investigation phase in sexual crimes allegations where the alleged victims were children and adolescents. The use of protocols, the profession of persons responsible for conducting the interviews, styles to start the interviews, the strategies to build rapport, the strategies to assess the skills of those interviewed as witnesses and the strategies to assess suggestibility were analyzed in the study. In general, it was found that during the interviews, the practices do not meet the suggested procedures stablished in the guidelines and protocols; therefore, mistakes -that are supposed to be rectified with these guidelines and protocols- are made.
\end{abstract}

\section{c) (i) $९$}

Referencia de este artículo (APA):

Gutiérrez de Piñeres, C. (2017). Análisis de las prácticas de entrevistas forenses durante la etapa de investigación por denuncias de delitos sexuales en contra de niños, niñas y adolescentes. Psicogente, 20(37), 118-134. http://doi.org/10.17081/psico.20.37.2422

Proyecto Análisis de las prácticas en las entrevistas judiciales que realizan los psicólogos en la etapa de investigación por denuncias de actos sexuales en contra de niños, niñas, financiado por la Fundación Universitaria Konrad Lorenz.

1. Doctor en Psicología con Orientación en Neurociencia Cognitiva Aplicada, Docente, Facultad de Psicología, Fundación Universitaria Konrad Lorenz. Email: carolinagdep@yahoo.com http://orcid.org/0000-0001-9079-3670 


\section{Introducción}

En Colombia, las entrevistas forenses realizadas durante la etapa de investigación en casos de denuncias por delitos sexuales, cuando se presume que la víctima ha sido un niño, niña o adolescente, son consideradas como un elemento material probatorio y tienen como objetivo principal reunir información precisa que oriente la investigación penal (Saywitz, Lyon \& Goodman, 2011) y que sirva, posteriormente, para orientar decisiones de índole judicial.

Las particularidades que reviste este delito, como la edad de las presuntas víctimas y su nivel de desarrollo cognitivo y emocional, así como las condiciones de intimidad bajo las cuales se desarrollan este tipo de hechos, las cuales hacen que en muchos casos no exista otra evidencia que corrobore lo dicho por la víctima, conducen a que sea más complejo obtener información pertinente para la investigación. Por esta razón, existe un consenso sobre la utilización de guías o protocolos de entrevistas forenses (American Professional Society on the Abuse of Children, 1990; Lamb, Sternberg \& Esplin, 1994; Raskin \& Esplin, 1991; Cantón \& Cortés, 2008), los cuales, combinando una serie de preguntas, fases y pasos, buscan que se realicen entrevistas de una manera más eficaz. Con la utilización de dichas guías y protocolos se busca cumplir dos objetivos: por un lado, proteger a los niños y adolescentes que resulten víctimas, al mismo tiempo que se protegen los derechos de las personas inocentes que hayan quedado vinculadas a un proceso penal por una falsa denuncia. Por otro lado, el desarrollo y uso de guías o protocolos pretende que se eliminen preguntas sugestivas que afecten la exactitud de la declaración (Poole \& Lamb, 1998); se incremente la fidelidad en los recuerdos de los testigos; se facilite a los investigadores la realización de entrevistas que tengan un menor im- pacto sobre la memoria original del evento; se reduzca el número de entrevistas y se ajusten a las características particulares de cada niño o adolescente de manera que se garantice su interés superior, al tener en cuenta las diversas habilidades comunicativas, el conocimiento social y las características de la memoria de cada uno de los entrevistados (Lamb, Orbach, Hershkowitz, Esplin \& Horowitz, 2007).

Tradicionalmente, se ha encontrado que las entrevistas realizadas a niños y adolescentes que se presume han sido víctimas de delitos sexuales contienen una serie de preguntas e información que pueden afectar el recuerdo de forma que al pasar el tiempo sea imposible recordar con precisión detalles del evento original, o pueden generar falsos recuerdos. Por ejemplo, se ha encontrado que la realización de preguntas asociadas semánticamente pueden generar un falso recuerdo (Curran, Schacter, Johnson \& Spinks, 2001); que la realización de entrevistas cuando ha pasado mucho tiempo puede afectar el recuerdo original (Loftus, 1979); que las personas que son menos exactas cuando relatan un evento son más susceptibles a la sugestión generada a través de información engañosa presentada después del evento (Loftus, Levidow \& Duensing, 1992) y que las preguntas dirigidas como: “¿El vestido que llevabas puesto era azul?", cuando el menor no había mencionado un vestido azul, pueden hacer que el niño incorpore información imprecisa en su relato personal (Perona, Bottoms \& Sorenson, 2006; Poole \& Lamb, 1998; Goodman \& Aman, 1990).

Del mismo modo, Bow, Quinnell, Zaroff y Assemany (2002) han mostrado cómo las evaluaciones y entrevistas que realizan los psicólogos en situaciones de denuncias por delitos sexuales, pocas veces se ciñen a los protocolos, guías o modelos de evaluación. Ceci y Bruck (1995) han evidenciado cómo cierto tipo de entrevistas 
incrementan el riesgo de contaminar el recuerdo de las presuntas víctimas, acrecentándose el número de errores de comisión más que de omisión. Sachsenmaier y Watson (1998) y Coulborn (2005) han señalado algunos errores en las entrevistas que afectan la declaración del niño o niña y favorecen la creación de falsas memorias como: a) preguntar al niño si lo que dice es verdad o mentira sin tener en cuenta que, especialmente en los niños más pequeños, no diferencian entre la verdad y la mentira; b) creer que si el niño dice que fue abusado sexualmente está diciendo la verdad; c) la utilización de muñecos anatómicos o láminas anatómicas que, al no asemejarse a nada que el niño haya visto en la realidad, pueden generar en algunos casos emociones fuertes o pueden despertar curiosidad y plantar nuevos recuerdos; d) utilizar preguntas cerradas, de respuesta si/no, o que sugieran respuestas; e) atribuir ciertas conductas como causas de abuso sexual; f) no estar atento a los propios sesgos; g) prestar atención únicamente a los datos que confirman el abuso; h) creer que los niños nunca mienten; i) entrevistar a los niños en varias oportunidades y por personas siempre diferentes; $j$ ) no grabar o filmar la entrevista y la evaluación, y k) mezclar la evaluación psicológica forense con la evaluación psicológica clínica.

Para subsanar los errores citados anteriormente, los protocolos y guías de entrevista forense como la entrevista cognoscitiva (Fisher \& Geiselman, 1992), la entrevista paso a paso (Yuille, Hunter, Joffe \& Zaparniuk, 1993), la guía de Poole y Lamb (Poole \& Lamb, 1998), la elaboración narrativa (Saywitz \& Snyder, 1996), el protocolo del Center for Child Protection (Center for Child Protection, 1973), el protocolo del National Children's Advocacy Center (Carens, Wilson \& Nelson, 1999), el protocolo del NICHD (Lamb et al., 2007), y el RATAC-SATAC (Anderson et al., 2010), coinciden en que las entrevistas en estos casos deben seguir al menos las siguientes indicaciones: a) las entrevistas deben realizarse lo más cercanas en tiempo a la denuncia; b) debe construirse rapport con el entrevistado; c) deben utilizarse estrategias que permitan al entrevistador conocer la capacidad del entrevistado para dar una declaración o aportar un testimonio (atención, memoria, percepción, pensamiento, desarrollo del lenguaje, capacidad de comprensión, metamemoria y metacognición y nivel de sugestionabilidad); d) debe hacerse una introducción al tema que será tratado en la entrevista; e) deben hacerse preguntas que faciliten un recuerdo libre; f) posterior al relato libre, deben hacerse preguntas que aclaren los puntos que generaron confusión; g) deben ajustarse los tiempos y la manera de preguntar a la edad y desarrollo de cada entrevistado (Cederborg et al., 2000; Lamb et al., 2007; Anderson et al., 2010; Walters, Holmes, Bauer $\&$ Vieth, 2003).

Asimismo, por tratarse de niños, niñas y adolescentes es importante considerar que deben observarse y atenderse una serie de aspectos psicológicos que podrán tener un efecto sobre la declaración de la presunta víctima y por lo tanto sobre el recuerdo del hecho. Por ello, en general, las guías y protocolos coinciden en sugerir que las entrevistas: a) deben iniciarse con estrategias de rapport y empatía y b) deben incluir la evaluación de competencias cognitivas según la edad del entrevistado: una evaluación sobre la comprensión de la diferencia entre la verdad y la mentira, una evaluación que explore capacidades de atención, memoria, percepción, pensamiento, desarrollo del lenguaje (capacidad de comprensión), metamemoria y metacognición y una evaluación sobre el nivel de sugestionabilidad (Ceci \& Bruck, 1995).

Vale la pena señalar, además de lo anterior, que las distintas guías o protocolos están centradas en el bienestar y el interés superior del entrevistado por tratar- 
se en estos casos de niños, niñas o adolescentes, quienes para la ley y la sociedad son poblaciones que merecen una protección especial por estar en una situación de vulnerabilidad. Lo anterior implica, entre otras cosas, que los entrevistadores deben ser personas altamente preparadas y especializadas, de forma tal que garanticen la integridad y el bienestar de los entrevistados (Berlinerblau, Nino \& Viola, 2013; UNICEF, 2003) y se disminuya la posibilidad de aparición de un daño iatrogénico (Di Lorenzo \& García, 2010).

Adicionalmente, en Colombia la ley y la jurisprudencia establecen que las entrevistas forenses sean practicadas por la Policía Judicial siguiendo reglas orientadas sobre lineamientos y disposiciones como las que se presentan a continuación: a) cuando se realiza una entrevista se debe informar, según el artículo 385 del Código de Procedimiento Penal, sobre las excepciones constitucionales que se expresan en el artículo 33 de la Constitución Política colombiana: "Nadie podrá ser obligado a declarar contra sí mismo o contra su cónyuge, compañero permanente o parientes dentro del cuarto grado de consanguinidad, segundo de afinidad o primero civil”. Esta aclaración debe hacerse a los padres o tutores del niño, niña o adolescente a quien se le realizará la entrevista, o al adolescente cuando este tenga más de 14 años; b) la entrevista debe ser fijada y dicho registro debe someterse a una cadena de custodia (Bedoya, 2008); c) al ser un acto de investigación, se practica antes de la formulación de afirmaciones propias de los actos de prueba y se refiere a una hipótesis sobre los hechos que permitirá luego hacer afirmaciones (Ortells, 1998); d) pueden desarrollarse con holgura porque no están condicionadas por las afirmaciones iniciales sobre la ocurrencia de unos presuntos actos delictivos (Ortells, 1998); e) hacen parte de la etapa de indagación y de investigación y por ello deben someterse a las funciones propias de estas etapas, que incluyen, entre otras cosas, las imputaciones y las medidas cautelares (Ortells, 1998); f) funcionan como acreditación y conocimiento sumario, por ello su eficacia jurídica no recae sobre la convicción plena de la responsabilidad de una persona; g) tienen como fin preparar el proceso a través de la"recaudación y obtención de las evidencias físicas o los elementos materiales probatorios que serán utilizados en el juicio oral para verificar las proposiciones de las partes y el Ministerio Público y, para justificar, con grado de probabilidad, las decisiones que corresponden al juez de control de garantías en las etapas preliminares del procedimiento" (Sentencia C-144/10) de la Corte Constitucional colombiana; h) se enmarcan en el seno de la instrucción; i) tienen por misión introducir los hechos en el procedimiento y contribuir a formar en el juez el juicio de probabilidad suficiente para disponer la imputación y adoptar las oportunas medidas cautelares, pero para que el juez tome una decisión debe haberse basado en actos de prueba; j) "se adelantan por la Fiscalía, la Defensa, el Ministerio Público y la víctima con el control y vigilancia del juez de control de garantías" (Sentencia C-144/10); k) en el caso de la Fiscalía, es el fiscal quien ordena la realización de los actos de investigación que no impliquen restricción de derechos fundamentales (Manual de Policía Judicial colombiana); 1) en el caso colombiano la investigación no se agota con la formulación de imputación, por lo tanto, los actos de investigación incluso pueden practicarse durante el juicio por previsión expresa del inciso final del artículo 344: “...si durante el juicio alguna de las partes encuentra un elemento material probatorio y evidencia física muy significativo que debería ser descubierto, lo pondrá en conocimiento del juez quien, oídas las partes y considerado el perjuicio que podría producirse al derecho de defensa e integridad del juicio, decidirá si es excepcionalmente admisible o si debe excluirse esa prueba (sic)" (Manual de Policía Judicial Colombiana, 
p.32); m) generalmente los actos de investigación son precedidos por el programa metodológico cuando se trata de la Fiscalía; n) según Montero (1991), en la medida en que los actos de investigación se documentan, cabría pensar, en principio, que las entrevistas podrían ser consideradas una fuente de prueba documental, es decir, que pueden acceder al juicio oral a través del medio de prueba documental, por ello su admisión deberá ser necesariamente restrictiva, porque en caso contrario se corre el riesgo de que los actos de prueba ante el tribunal sentenciador sean sustituidos por aquellos documentos, con infracción de los principios del juicio oral; o) "las autoridades judiciales que intervengan en las etapas de investigación y juzgamiento de delitos sexuales cometidos contra menores deben abstenerse de actuar de manera discriminatoria contra las víctimas, estando en la obligación de tomar en consideración la situación de indefensión en la cual se encuentra cualquier niño que ha sido sujeto pasivo de esta clase de ilícitos. De tal suerte, que constituyen actos de discriminación cualquier comportamiento del funcionario judicial que no tome en consideración la situación de indefensión en la que se encuentra el menor abusado sexualmente, y por lo tanto dispense a la víctima el mismo trato que regularmente se le acuerda a un adulto, omita realizar las actividades necesarias para su protección, asuma una actitud pasiva en materia probatoria, lo intimide o coaccione de cualquier manera para que declare en algún u otro sentido o para que no lo haga. Tales prácticas vulneran gravemente la Constitución y comprometen la responsabilidad penal y disciplinaria del funcionario que las cometa" (Sentencia T-078, 2010 de la Corte Constitucional colombiana, p.2); p) deben practicarse en cámara de Gesell o en su defecto en un espacio adecuado que contemple la edad y la etapa de desarrollo de la presunta víctima (Ley 1652 de 2013); q) como resultado de la entrevista, de acuerdo con el artículo 209 de la Ley 906, debe realizarse un informe detallado que contenga: una descripción clara y precisa de la forma, técnica e instrumentos utilizados en la actividad investigativa a que se refiere el informe; una descripción clara y precisa de los resultados de la actividad investigativa antes mencionada; una relación clara y precisa de los elementos materiales probatorios y evidencia física descubiertos, así como de su recolección, embalaje y sometimiento a cadena de custodia; r) debe quedar registro de la entrevista en medio magnético; s) debe realizarse preferiblemente una única entrevista; t) el acceso a las mismas tendrá ciertas restricciones y solo se podrá acceder a ellas cuando sea estrictamente necesario, respetando los derechos de la víctima (Ley 1652 de 2013); u) la entrevista no debe seguir necesariamente un protocolo (Proceso 31950 de 2009 de la Corte Suprema de Justicia), no obstante en sus procedimientos la Fiscalía General de la Nación opta por aplicar el protocolo SATAC-RATAC y v) las entrevistas deben seguir siempre el principio del interés superior del menor (Sentencia T-117/13).

A partir de lo anterior, la presente investigación se enfocó en analizar las prácticas en las entrevistas forenses que se realizan en Colombia durante la etapa de investigación por denuncias de delitos sexuales en las que se presume han sido víctimas niños, niñas o adolescentes. Se espera que los hallazgos aquí presentados sirvan para mejorar los procedimientos utilizados en estas entrevistas y que se motive a los funcionarios que las realizan a cualificarse más con el fin de garantizar el interés superior del niño y colaborar de esta forma con una humanización de la justicia.

\section{Método}

\section{Diseño}

Se realizó un estudio cualitativo con un diseño de teoría fundamentada (Hernández, Fernández \& Bap- 
tista, 2010; Sandoval, 1996), con perspectiva explicativa (Sandoval, 1996), con enfoque del interaccionismo simbólico que surge de las teorías de la cultura y que utiliza un análisis de contenido (Strauss, 1987).

El método utilizado para el análisis de datos fue un análisis de contenido de tipo categorial. La herramienta utilizada fue Excel.

\section{Unidades de análisis}

Las unidades de análisis fueron las prácticas ("actividad continua, definida por los miembros de un sistema social como rutinaria”) (Hernández, Fernández \& Baptista, 2010) en las entrevistas realizadas por los investigadores de Policía Judicial del Cuerpo Técnico de Investigación (CTI) durante las entrevistas efectuadas a presuntas víctimas de delitos contra la libertad, integridad y formación sexual. Solo se analizaron las preguntas y las estrategias durante el desarrollo de las entrevistas, pero no las respuestas que daban los niños.

La fuente de recolección de datos cualitativos fueron informes y transcripciones de entrevistas realizadas durante la etapa de investigación.

\section{Participantes}

Se analizaron 81 entrevistas realizadas por la Policía Judicial colombiana durante la etapa de investigación en denuncias por delitos sexuales y un total de 2.789 preguntas. De los casos analizados, las presuntas víctimas fueron 9 del sexo masculino y 72 del sexo femenino. Las edades de los niños entrevistados oscilaban entre los 3 y los 17 años de edad. Los lugares de ocurrencia y realización de las entrevistas fueron: 51 en Bogotá, 8 en el Eje Cafetero, 8 en Cundinamarca, 3 en el Tolima y 11 en otras regiones. Para la selección de las entrevistas se tuvo en cuenta que fueran realizadas durante la etapa de investigación del proceso penal, por Policía Judicial del Cuerpo Técnico de Investigación (CTI) de la Fiscalía General de la Nación (FGN) a niños que habían sido presuntas víctimas y que se encontraban entre los 3 y los 17 años y 11 meses, en casos de denuncias por delitos contra la libertad, integridad y formación sexual. Se excluyeron las entrevistas realizadas por defensores de familia, las realizadas por ONG o por psicólogos del Instituto Colombiano de Bienestar Familiar, las entrevistas que se realizaron en otras etapas del proceso penal y las que se realizaron a testigos.

\section{Instrumentos}

Se diseñó una tabla en Excel con las categorías a partir de las cuales se clasificaría la información recolectada en las entrevistas. Las categorías se obtuvieron a partir de los protocolos y guías que se utilizan con mayor frecuencia en este tipo de entrevistas, como el SATACRATAC, el protocolo de Michigan, el NICHD, la entrevista cognoscitiva, la entrevista paso a paso, la guía de Poole y Lamb, el protocolo del Center for Child Protection y el memorándum de la buena práctica.

En la Tabla 1 se presenta el árbol categorial que sirvió de base para el análisis de las entrevistas.

\section{Tabla 1. Árbol categorial}

\begin{tabular}{ll}
\hline \multicolumn{1}{c}{ Categoría } & \multicolumn{1}{c}{ Definición } \\
\hline $\begin{array}{l}\text { Reporte de uso de } \\
\text { protocolos o guías }\end{array}$ & $\begin{array}{l}\text { Las guías y protocolos de entrevista son estrategias } \\
\text { de entrevista }\end{array}$ \\
& rentes organismos, que combinan preguntas, fases \\
& y pasos, buscando que se realicen entrevistas de una \\
& manera más eficaz y eficiente (American Professio- \\
& nal Society on the Abuse of Children, 1990; Lamb, \\
& Sternberg \& Esplin, 1994; Raskin \& Esplin, 1991; \\
& Poole \& Lamb, 1998). \\
\hline
\end{tabular}




\begin{tabular}{|c|c|}
\hline Categoría & Definición \\
\hline Entrevistadores & $\begin{array}{l}\text { Deben ser personas capacitadas, preferiblemente } \\
\text { profesionales en Psicología, con un entrenamiento } \\
\text { específico en la realización de entrevistas en casos } \\
\text { de denuncias por delitos sexuales. }\end{array}$ \\
\hline $\begin{array}{l}\text { Estilos para iniciar } \\
\text { la entrevista }\end{array}$ & $\begin{array}{l}\text { De acuerdo con Echeburúa, Amor y Corral (2003), } \\
\text { los estilos para iniciar la entrevista hacen parte de } \\
\text { una de las fases más importantes. Durante esta fase } \\
\text { se establece una relación adecuada con el entrevis- } \\
\text { tado, se recoge información general, se hace una } \\
\text { presentación de la persona que realiza la entrevista, } \\
\text { se presentan las reglas generales de la entrevista, se } \\
\text { explican los derechos del entrevistado adaptados a } \\
\text { su edad y desarrollo evolutivo, se aclaran los proce- } \\
\text { dimientos y las herramientas que serán utilizadas y } \\
\text { se hace una descripción del lugar donde se realiza } \\
\text { la entrevista. }\end{array}$ \\
\hline Rapport y empatía & $\begin{array}{l}\text { El rapport es la forma en la que se establece un am- } \\
\text { biente de confianza antes de comenzar a indagar por } \\
\text { el tema del presunto abuso. } \\
\text { La empatía se refiere a la capacidad del entrevista- } \\
\text { dor para ponerse en el lugar del entrevistado, to- } \\
\text { mando conciencia de las diferencias evolutivas y las } \\
\text { competencias como testigo del entrevistado. }\end{array}$ \\
\hline $\begin{array}{l}\text { Evaluación de } \\
\text { competencias del } \\
\text { entrevistado como } \\
\text { testigo }\end{array}$ & $\begin{array}{l}\text { Busca identificar las competencias del entrevistado } \\
\text { como testigo, indagando por procesos como la aten- } \\
\text { ción, la percepción, la memoria, la metamemoria y } \\
\text { otros procesos metacognitivos, desarrollo del len- } \\
\text { guaje, su capacidad para diferenciar entre un relato } \\
\text { verdadero y uno falso, su capacidad para razonar, } \\
\text { el conocimiento que el entrevistado tiene sobre las } \\
\text { partes del cuerpo, la formación que el entrevistado } \\
\text { ha recibido sobre educación sexual y la competen- } \\
\text { cia del entrevistado para reportar experiencias de } \\
\text { toques positivos y negativos. }\end{array}$ \\
\hline $\begin{array}{l}\text { Evaluación de la } \\
\text { sugestionabilidad }\end{array}$ & $\begin{array}{l}\text { Se refiere a la evaluación del impacto que tienen } \\
\text { diferentes factores, eventos y personas sobre la co- } \\
\text { dificación, almacenamiento, recuperación y relato } \\
\text { de un hecho (Juárez, 2006; Gudjonsson \& Clark, } \\
\text { 1986). }\end{array}$ \\
\hline
\end{tabular}

\section{Procedimiento}

Primera fase: Se seleccionaron las entrevistas de acuerdo con los criterios de inclusión y exclusión. Se hizo una transliteración de las entrevistas ya que todas se encontraban grabadas en audio y luego se leyó al menos dos veces cada una.
Segunda fase: Se diseñó el árbol categorial y se completó con las categorías emergentes que surgían a partir de la lectura repetida de las entrevistas. Se codificaron los datos según las categorías. Posteriormente, se hizo un examen a la manera en que habían sigo categorizadas las preguntas y las estrategias utilizadas por los entrevistadores y se recategorizaron de acuerdo con este análisis.

Tercera fase: Se realizó el análisis de acuerdo con cada una de las categorías, se elaboró la discusión y las conclusiones.

\section{Resultados}

\section{Reporte de uso de protocolos o guías de entrevista}

De los 81 casos analizados, solo en 33 se reportó el tipo de protocolo, guía o entrevista utilizada. De estas, en 15 se reportó el uso del SATAC, en 4 el NICHD, en 4 la entrevista cognitiva y en 8 otro tipo de protocolos, guías o entrevistas sin especificar cuál se utilizó.

\section{Entrevistadores}

Dentro de las personas que firmaban como responsables de la realización de la entrevista, se encontraban 38 psicólogos, 16 investigadores criminalistas, 15 policías judiciales, 4 defensores de familia, 1 asistente de fiscal y 7 firmaron sin describir su cargo o función. Es posible que fueran psicólogos quienes firmaron como investigadores criminalistas o como policía judicial, pero no se especifica en estos casos.

\section{Estilos utilizados para iniciar las entrevistas}

La Tabla 2 muestra algunas de las estrategias utilizadas para dar inicio a las entrevistas. 
Tabla 2. Estilos utilizados para iniciar las entrevistas

\section{Categoría 1 Temas, estrategiasy preguntas relacionadas}

Estilos para iniciar 1. Indagar datos familiares y académicos o datos perla entrevistas sonales como la edad y el nombre.

2. Utilizar preguntas para romper el hielo con el entrevistado, por ejemplo: ¿cómo te gusta que te llamen? ¿Qué es lo que más te gusta hacer? O hacer una presentación personal del funcionario que realiza la entrevista.

3. Señalar la importancia de decir la verdad o señalar que se indaga si el entrevistado comprende la diferencia entre el concepto de verdad y mentira, pero en algunos casos no se describe cómo se realiza este proceso. Este aspecto se indaga con preguntas como: soy psicóloga, trabajo aquí en la Comisaría de Familia, parte de mi trabajo es hablar con niñas que me han contado verdades sobre cosas que les han sucedido y siempre me han dicho la verdad; si yo te digo, por ejemplo, que las vacas vuelan, ies verdad o mentira?; si te digo que mis zapatos son de color rojo, ¿qué dirías?; si yo te digo que tú te llamas Juan Pablo, ¿es verdad o es mentira?; si yo te digo que el cielo es rojo, ¿esto sería cierto o falso?

4. Indagar acerca del conocimiento que tiene el entrevistado sobre las partes del cuerpo y los nombres que utiliza para denominarlas. Se utilizan preguntas como: ¿yo soy hombre o mujer? Indícanos ¿cuáles son las partes de tu cuerpo incluyendo tus partes íntimas? ¿Tú conoces las partes del cuerpo? Hay partes en el cuerpo que se llaman partes íntimas, ¿con qué nombre conoces esas partes intimas?

5. En algunas transcripciones de las entrevistas se menciona que se da inicio a las mismas propiciando un ambiente de empatía, pero no se describe cómo se hace.

6. Se señalan los derechos establecidos en el artículo 385 del Código de Procedimiento Penal, sobre las excepciones constitucionales. Se utilizan preguntas como: ¿entiendes tus derechos?

7. Se indaga sobre el conocimiento que tiene el niño acerca del motivo de la entrevista, con preguntas como: ¿sabes por qué estás hoy aquî? ¿conoce usted el motivo de la presente diligencia? ¿sabes el motivo por el que nos encontramos el día de hoy acá en este recinto? Sírvase informar si conoce el motivo por el cual fue citada. Cuéntele a esta defensora de familia si sabe el motivo por el cual está dando esta entrevista.

8. Se indaga directamente por la situación que motiva la denuncia, con preguntas como: ¿cuéntame qué te pasó? ¿A ti te ha pasado algo alguna vez en tu cuca, tus senos o en tu cola? ¿Quiero que me cuentes todo lo que pasó ese día?

\begin{tabular}{ll}
\hline Categoría 1 & \multicolumn{1}{c}{ Temas, estrategiasy preguntas relacionadas } \\
\hline & 9. Se indaga sobre los toques y tactos, con preguntas \\
como: ite han dado besos, abrazos o caricias que no & te gusten o que te incomoden? \\
& 10. Se evalúan aspectos relacionados con la orienta- \\
& ción. Para esto se utilizan preguntas como: ¿qué día \\
& es hoy? En algunos casos se dice que se evalúan los \\
& procesos cognitivos, pero no se describe la forma en \\
& que se realiza dicha evaluación. \\
\hline
\end{tabular}

Se observa que no existe una única forma para dar inicio a la entrevista y que en la mayoría de casos no se cumple con los lineamientos establecidos por la ley o con los sugeridos en los protocolos y guías de entrevistas utilizados tradicionalmente en las denuncias por delitos sexuales contra niños, niñas o adolescentes.

De las 81 entrevistas analizadas solo en un caso se menciona haberle dicho al entrevistado sobre su derecho a no declarar, según lo establecido en el artículo 385 del Código de Procedimiento Penal colombiano. Solo en algunos casos se encuentra que se les haya explicado a los entrevistados el motivo de la entrevista y, aunque en casi todos se le pregunta al entrevistado si conoce la razón por la cual se le está realizando la entrevista, en ningún caso se menciona o explica su objetivo. En ningún caso se encuentra que se le haya explicado al entrevistado el uso de la cámara de Gesell. En la mayoría de casos se utilizan estrategias para romper el hielo, pero se evidencia mayor dificultad en la iniciación de las entrevistas en aquellas personas que no cuentan con el título de Psicología. Asimismo, se evidencia el uso de una gran cantidad de preguntas cerradas, el uso de preguntas sugestivas y directivas.

\section{Rapport y empatía}

En la Tabla 3 se presenta una lista de las principales estrategias de rapport utilizadas por los entrevistadores. 
Tabla 3. Rapport y empatía

\begin{tabular}{|c|c|}
\hline Categoría 2 & Temas, estrategias y preguntas relacionadas \\
\hline $\begin{array}{l}\text { Rapport y } \\
\text { empatía }\end{array}$ & $\begin{array}{l}\text { Preguntar por la relación con la familia. } \\
\text { Preguntar por los miembros que conforman la familia. } \\
\text { Presentación personal de la persona que realiza la en- } \\
\text { trevista. } \\
\text { Preguntar al entrevistado cómo le gusta que lo llamen. } \\
\text { Preguntar al entrevistado qué le gusta hacer o sobre lo } \\
\text { que hace. }\end{array}$ \\
\hline
\end{tabular}

En la mayoría de informes se reporta haber implementado alguna estrategia orientada al desarrollo del rapport y la empatía, tal y como puede verse en la Tabla 3 , pero se evidencia una ausencia en la descripción de la forma en que se lleva a cabo esta etapa. En ningún caso se observan como estrategia para el desarrollo de la empatía las propuestas en el protocolo SATAC, tales como el dibujo de una cara o de los círculos de la familia, pese a que en 15 de las entrevistas analizadas se reporta el uso de este protocolo.

\section{Evaluación de competencias del entrevistado como testigo}

En la Tabla 4 se presenta una lista con algunas de las preguntas utilizadas por los entrevistadores para evaluar las competencias de los entrevistados como testigos. Se evidencia que en todos los casos la única herramienta para realizar esta evaluación es la entrevista misma. En ningún caso se reporta el uso de test psicológicos.

Tabla 4. Evaluación de competencias del entrevistado como testigo

\begin{tabular}{ll}
\hline Categoría 3 & Temas, estrategias y preguntas relacionadas \\
\hline Evaluación de & Capacidad para diferenciar entre un relato verdadero y \\
competencias & uno falso. Se evaluó a través de preguntas u oraciones \\
del entrevista- & como: Como quiero estar segura de que me estás enten- \\
do como tes- & diendo y me estás prestando atención, te voy a preguntar \\
tigo & una cosa: si te digo que mis zapatos son de color rojo, \\
& ¿qué dirías? Si te digo que mi cabello es mono, itú qué \\
& dirías? :Entonces tú sabes qué es mentir y qué es decir la \\
& verdad? ¿Sabes qué es decir la verdad?
\end{tabular}

\begin{tabular}{|c|c|}
\hline Categoría 3 & Temas, estrategias y preguntas relacionadas \\
\hline & $\begin{array}{l}\text { Capacidad del entrevistado para relatar datos de su vida. } \\
\text { Se evaluó a través de preguntas como: ¿Díganos cuál es } \\
\text { la fecha de su nacimiento? ¿Díganos con qué personas } \\
\text { vive usted? Sírvase informar nombre completo, edad, } \\
\text { estudios, padres y convivencia familiar. ¿Cuántos años } \\
\text { tienes? ¿Cómo se llama tu colegio? ¿Cómo se llama tu } \\
\text { mamá? } \\
\text { Conocimiento que el entrevistado tiene sobre las partes } \\
\text { del cuerpo, a través de preguntas como: ¿Tú conoces las } \\
\text { partes del cuerpo? Entonces yo te voy a ir señalando al- } \\
\text { gunas partes y tú me vas diciendo cuál es el nombre de } \\
\text { esas partes ¿listo? ¿Tú sabes cuáles son las partes privadas, } \\
\text { cuáles? } \\
\text { La competencia del entrevistado para reportar experien- } \\
\text { cias de toques positivos y negativos, a través de preguntas } \\
\text { como: ¿Te han dado besos, abrazos o caricias que no te } \\
\text { gusten o que te incomoden? } \\
\text { Capacidad para reportar escenario de abuso, a través de } \\
\text { preguntas como: Empecemos por saber el nombre de esa } \\
\text { persona. ¿Qué pasó con esa persona? ¿Cuáles partes ínti- } \\
\text { mas te tocó esa persona? ¿Y esto fue por encima o por de- } \\
\text { bajo de tu ropa? ¿Cuántas veces pasó? Háblame de la pri- } \\
\text { mera vez. Cuando él te metió el pene por la cola itú qué } \\
\text { sentiste? ¿Tú me podrías decir si en algún momento ha } \\
\text { pasado algo en alguna de las partes de tu cuerpo? ¿Esas } \\
\text { personas estaban con ropa o sin ropa? Me puedes contar } \\
\text { específicamente, quiero que me cuentes específicamente } \\
\text { lo que pasó en tu boca y en tu vagina, cuéntame deta- } \\
\text { lladamente. Tú me dices dónde estaban cuando pasó } \\
\text { esa situación. Sírvase decir que sucedió el..., relatando } \\
\text { pormenorizadamente las actividades sexuales abusivas } \\
\text { que realizó el agresor contra usted, ubicando el sitio, la } \\
\text { hora, cómo ocurrió el abuso, si fue repetitivo, qué actos } \\
\text { fueron realizados por parte del denunciado. Igualmen- } \\
\text { te, establecer las maniobras y palabras sexuales que este } \\
\text { sujeto le manifestó, si hubo algún tipo de violencia o } \\
\text { amenaza durante el abuso, si utilizó algún tipo de arma } \\
\text { o promesa, remuneración u otro incentivo. ¿Qué sucedía } \\
\text { en ese momento, qué era lo que hacía él? Ok, muy bien, } \\
\text { si en algún momentes privadas, ahora itú me podrías decir } \\
\text { parterpo? }\end{array}$ \\
\hline
\end{tabular}

Como se describe en la Tabla 4, la principal técnica para indagar acerca de la capacidad del niño para diferenciar entre una verdad y una mentira es preguntándole por la forma o color de un objeto contrario a lo que ocurre en la realidad. Para explorar la capacidad del entrevistado para reportar datos personales y familiares 
se utilizan preguntas generales combinando preguntas abiertas y cerradas. Asimismo, se aprecia en algunos casos la utilización de un lenguaje poco empático para comunicarse con niños, niñas o adolescentes, como lo muestra el siguiente ejemplo: "Sírvase informar nombre completo, edad, estudios, padres y convivencia familiar; ¿cuántos años tienes?”. Esta forma de preguntar además involucra varias preguntas en una misma oración. Para explorar el conocimiento que el niño tiene sobre las partes del cuerpo se utilizan en la mayoría de casos dos láminas, una con una figura femenina y otra con una figura masculina.

Para explorar los reportes de experiencias de toques positivos y negativos y examinar la capacidad de los niños para reportar un escenario de abuso, se utilizan ejemplos cotidianos en los que se relatan diversas situaciones de toques entre personas. Asimismo, se observa la utilización de preguntas sugestivas, pues se indaga por hechos puntuales que el entrevistado previamente no ha reportado. En algunos casos las preguntas parten de la hipótesis según la cual los hechos ocurrieron y no se hacen preguntas posteriores que indaguen una hipótesis contraria. Se hacen preguntas que pueden resultar complejas para la edad del entrevistado, a través de las cuales se indaga sobre su percepción acerca de las intenciones que presuntamente podría tener la persona que fue denunciada, sin indagar previamente los procesos de neurodesarrollo.

\section{Evaluación de la sugestionabilidad}

En ninguna entrevista se encuentra alguna estrategia para evaluar la predisposición a la sugestionabilidad.

\section{Discusión y Conclusiones}

Los hallazgos de esta investigación son consisten- tes con otros estudios similares centrados en el análisis de las prácticas de evaluación y entrevista en casos de delitos sexuales, procesos de custodia y maltrato infantil (Herman, 1997; Ceci \& Bruck, 1993; Lamb et al., 2000; Bow \& Quinnell, 2001a; Bow \& Quinnell, 2001b; Bow $\&$ Boxer, 2003). En general, se evidencia que las entrevistas no se ajustan a ninguno de los protocolos sugeridos; que se cometen errores en la forma en que se plantean las preguntas, dándose una gran cantidad de preguntas sugestivas, inductivas y equívocas; que no se evalúan, o por lo menos no con los instrumentos adecuados, los procesos de neurodesarrollo que permitan identificar las competencias de los entrevistados como testigos; que no se siguen los lineamientos jurídicos; que pese a la importancia de indagar si el entrevistado es una persona sugestionable o no, no se encuentra en ninguna de las entrevistas analizadas una estrategia que haya sido utilizada para confirmar o rechazar la hipótesis de sugestionabilidad, es decir, no se indaga sobre la posibilidad de que factores individuales o contextuales hubieran podido afectar el relato del entrevistado; que en pocos casos se menciona el uso del consentimiento y del asentimiento informado; que la gran mayoría está enfocada en la hipótesis según la cual se presentó una situación de abuso y en pocos casos se realizan preguntas que confirmen hipótesis alternas u opuestas; que están centradas en el interés del entrevistador y no en el interés superior del niño; por citar algunos de los errores más comunes.

De otro lado, en cuanto a la forma de comenzar las entrevistas, en el caso de las entrevistas aquí analizadas se encontró que no se realiza un encuadre en el que se explique a los niños, niñas y adolescentes que participarán de las mismas, cómo se llevará a cabo la entrevista y cuáles serán las reglas que se seguirán. En pocas ocasiones se hace una presentación de la persona que realiza la entrevista, de los objetivos que se persiguen y de los derechos que tiene la persona que está 
siendo entrevistada a guardar silencio, a preguntar, si no tiene claridad en una pregunta, o a expresar su incomodidad sobre el desarrollo de la entrevista; así como de los aspectos relacionados con ciertos procedimientos como el uso de la cámara de Gesell o la grabación de la entrevista en audio o video. Asimismo, según se observa y escucha en los videos y audios, la forma en la que se da inicio a las entrevistas genera incomodidad e intimidación en el entrevistado, limitando la interacción de este con el entrevistador y reduciendo así la posibilidad de recopilar información suficiente para el desarrollo de cada caso. Esto contradice lo planteado por Anderson et al. (2010), quienes señalan que la manera en que se realizan las preguntas desde el inicio generará un impacto en la forma como los entrevistados responderán al resto de la entrevista. Para ello se sugiere, en la medida de lo posible, comenzar siempre con preguntas abiertas y solo realizar preguntas cerradas cuando las abiertas ya no brinden más información (Faller, 2005).

Este aspecto tiene un impacto directo sobre el rapport, que si bien se menciona como estrategia fundamental en la mayoría de entrevistas analizadas, no se evidencia específicamente la forma en que se desarrolla. En este sentido, es importante mencionar que el rapport es esencial cuando se trata de entrevistar a niños o adolescentes, más aún cuando se presume que estos han sido víctimas o testigos de algún delito. La utilización adecuada de estrategias para generar rapport incrementa la probabilidad de que los entrevistados respondan a la entrevista de la manera en la que el o los entrevistadores lo esperan (Anderson et al., 2010; Vallano \& Schreiber, 2011). Lo anterior no significa que el entrevistado responda lo que el entrevistador quiere escuchar, sino que el entrevistado sea más aquiescente y colaborador, de forma tal que se sienta relejado y motivado, y de esta forma brinde información más precisa (Collins, Lincoln \& Frank, 2002).
Vale la pena señalar que si bien el rapport en la entrevista forense es heredado de la entrevista clínica, en la cual se busca que el paciente o consultante se adhiera al tratamiento, en el ámbito de la entrevista forense esta estrategia, en ocasiones, puede generar cierta confusión, ya que el rapport es visto como una alianza con el entrevistado, lo cual puede conducir a una pérdida de objetividad en la recolección de la información, en la medida en que suele suceder que la forma en que se conducen estas entrevistas está orientada a confirmar la hipótesis según la cual un hecho de abuso sexual se presentó y no a analizar alternativas distintas como puede ser una conducta de aseo. Una pregunta que surge de la utilización del rapport en estas entrevistas es cómo equilibrar los objetivos que persigue la entrevista forense con los objetivos terapéuticos en caso de que se confirme una situación de abuso, teniendo presente que, aunque es importante mostrar sensibilidad ante la ocurrencia de un presunto delito sexual, la entrevista forense no tiene pretensiones terapéuticas sino el propósito de orientar una investigación y el de incrementar la exactitud y calidad en el recuerdo de unos presuntos hechos.

Las equivocaciones encontradas, a raíz de esta investigación, en la creación del rapport, pueden generar un impacto en el ambiente de confianza que debe crearse particularmente en estos casos y, a la vez, influir de manera negativa en la información que brinda el entrevistado.

Por lo anterior, se concluye que el rapport amerita que el entrevistador tenga varias habilidades. En primer lugar, una capacidad para demostrar empatía, es decir, capacidad de ponerse en el lugar del entrevistado (Grupo de Trabajo del Gobernador para la Justicia del Menor \& Agencia para la Independencia de la Familia, 2003). En segundo lugar, capacidad para comunicar simpatía, 
es decir, mostrarse amable y respetuoso frente al entrevistado, de forma honesta y sincera. Y en tercer lugar, capacidad para conseguir que el entrevistado relate aquellos hechos relevantes para la investigación (Anderson et al., 2010). Adicionalmente, teniendo en cuenta que las entrevistas que se realizan dentro de procesos judiciales no hacen factible que se den varios encuentros entre los entrevistados y los entrevistadores, a diferencia de lo que ocurre en otro tipo de entrevistas como las que se desarrollan dentro de un contexto clínico, es importante la habilidad del entrevistador para establecer rapport en los primeros momentos de la entrevista, utilizando para ello las técnicas sugeridas en los protocolos y guías ya establecidos, tales como dibujar, preguntar al niño su nombre y lo que hace, preguntarle al niño acerca de su familia o de su colegio, entre otras. Las técnicas sugeridas para establecer rapport, además de cumplir con funciones tales como servir de "rompe-hielo" o de lograr que el entrevistado se sienta cómodo, brindan una oportunidad para ir conociendo los estilos de comunicación del niño y sus habilidades para transmitir ideas.

De otro lado, teniendo en cuenta que la entrevista forense en estos casos tiene como una de sus pretensiones acceder al recuerdo de un hecho presuntamente ocurrido, por tratarse de niños y de adolescentes es primordial evaluar las competencias de los entrevistados para relatar un evento y los procesos de neurodesarrollo que pudieran tener un impacto sobre el recuerdo (Instituto Nacional de Medicina Legal y Ciencias Forenses, INMLCF, 2010; American Academy of Child and Adolescent Psychiatry, 1997). En este estudio se encontró que no se evalúan las competencias del testigo para dar un testimonio, aunque se utilizan algunas herramientas subjetivas para tener ciertos conocimientos particulares sobre este. Si bien es cierto que bajo las circunstancias adecuadas los niños y adolescentes son capaces de re- portar con exactitud los hechos relacionados con una posible situación de abuso, dicha capacidad está altamente influenciada por la forma en que se practica una entrevista y con variables personales, contextuales y de desarrollo. Por lo tanto, es necesario evaluar estas competencias a través de técnicas destinadas para tal fin. No obstante, ni los protocolos ni las guías indican el uso de estrategias objetivas y válidas para evaluar estas competencias. Algunos protocolos incluyen estrategias que facilitan indagar (mas no evaluar) sobre algunas de estas competencias, por ejemplo: pedirle al entrevistado que cuente un evento reciente como un cumpleaños o una rutina como ir a la cama o cenar. Sin embargo, este tipo de estrategias no contemplan el hecho de que el recuerdo de un evento se codifica de diferentes maneras, y esta codificación va a depender de varios factores como la edad del entrevistado, el valor emocional que el evento tuvo para él, la familiaridad con los hechos que motivan el relato, las competencias cognitivas (atención, memoria, aprendizaje, pensamiento, entre otras) y metacognitivas (metamemoria y teoría de la mente) del entrevistado, el lenguaje que se usa durante la entrevista, el tiempo que duró el evento, el número de veces que se repitió el evento, el tiempo transcurrido entre la ocurrencia del último episodio y la realización de la entrevista (Ceci \& Bruck, 1995; Carens, Wilson \& Nelson, 1999), de forma que, por ejemplo, no necesariamente porque un niño, niña o adolescente reporte con suficiente cantidad de detalles un evento que fue significativo, reportará con la misma destreza un presunto escenario de abuso, pues este último pudo haberse vivido como una situación cotidiana de aseo y por ello se limita el número de detalles que consigan ser recordados.

Finalmente, basado en el análisis de las transcripciones de las entrevistas realizadas a niños, niñas y adolescentes durante la etapa de investigación por denuncia 
de delitos sexuales, se puede concluir lo siguiente: en la mayoría de casos no se reporta que se le haya informado a los padres o a los entrevistados sus derechos; no se describe o menciona el lugar en el que se practicó la entrevista; existe una deficiencia en las estrategias de rapport y empatía para dar inicio a la entrevista; en la mayoría de entrevistas se incluyen preguntas a través de las cuales se busca evaluar la competencia del entrevistado para diferenciar entre verdad y mentira, pero en general las estrategias utilizadas solo permiten diferenciar la capacidad del niño para discriminar colores; no se incluyen estrategias para realizar evaluaciones que permitan identificar diferencias evolutivas o capacidades cognitivas; no se utilizan estrategias para evaluar el nivel de sugestionabilidad; no se practican preguntas que exploren alternativas diferentes frente a un presunto relato de abuso o inconsistencias en los relatos y, por el contrario, en algunos casos se asume desde el principio que los hechos ocurrieron, como se puede notar en el siguiente ejemplo: "Sírvase decir qué sucedió el XXXX, relatando pormenorizadamente las actividades sexuales abusivas que realizó el agresor contra usted, ubicando el sitio, la hora, cómo ocurrió el abuso, fue repetitivo, qué actos fueron realizados por parte del denunciado. Igualmente establecer las maniobras y palabras sexuales que este sujeto le manifestó, si hubo algún tipo de violencia, amenaza durante el abuso, asimismo utilizó algún tipo de arma o promesa, remuneración u otro incentivo”. Se realizan una gran cantidad de preguntas cerradas, dirigidas, ambiguas o sugestivas, sobre todo cuando no se tiene en cuenta el orden en el que se realizan las preguntas, por ejemplo: la entrevista comienza con la siguiente pregunta: " $i$ Te han dado besos, abrazos o caricias que no te gusten o que te incomoden?", a continuación se le pregunta al entrevistado: “iTú sabes porque estás aquí?". Otro ejemplo de este caso: el entrevistador pregunta: ¿Tú sabes cuáles son las partes privadas D?, ¿cuáles?, y a continuación se le pregunta: "Ok, muy bien, esas son las partes privadas ahora $\mathrm{D}$, dime ¿tú me podrías decir si en algún momento ha pasado algo en alguna de las partes de tu cuerpo?". Preguntas como estas se prestan para confusión ya que es posible que en diversos momentos el médico, su madre, una tía o un tío haya tocado estas partes a un niño, niña o adolescente, sin una intención sexual. También ocurre que se les pide a los entrevistados que realicen juicios de valor sin tener en cuenta su edad, por ejemplo: “¿Después de que pasó esa situación, has tenido malos sueños?”, “¿dar besos en la mejilla es malo?", "¿si te tocan las manos, las piernas o la cara, es malo?". En estos casos se desconoce que funciones cognitivas como la teoría de la mente, tan importantes para la comunicación y la interacción, se van haciendo más complejas con la maduración del sistema nervioso y por ello es tan importante conocer cuáles de estos procesos se han alcanzado y cuáles están en proceso de maduración, de manera que las entrevistas se puedan ajustar según su evolución.

Para concluir, se llama la atención sobre la importancia de mejorar la calidad y la rigurosidad en la aplicación de entrevistas forenses, ya que los procedimientos inadecuados pueden tener un efecto sobre los objetivos que se persiguen con las mismas. Por tanto, se hace necesario que se optimice la forma en que se elaboran las preguntas, que se sigan los lineamientos sugeridos en cada uno de los protocolos, que se estudien sus fundamentos propuestos y que las personas encargadas de realizar las entrevistas se actualicen permanentemente en la realización de entrevistas y en los procesos cognitivos y emocionales que subyacen al almacenamiento de información tanto semántica como episódica.

Los errores encontrados en la realización de las entrevistas pueden conllevar a que no se recupere información útil y precisa sobre la forma en que se desarrolló el presunto delito, la frecuencia y fechas en las que 
ocurrieron los hechos, la descripción de los lugares o información adicional que permita saber si el relato proviene de un hecho vivido, imaginado, inventado o sugerido; por lo tanto, una entrevista inadecuada no servirá como elemento material probatorio. En este punto es importante hacer la siguiente reflexión: ¿qué hace que el testimonio del entrevistador experto tenga más validez en un juicio y sea más confiable que el testimonio del padre, la madre o cualquier otro testigo que también haya tenido conocimiento indirecto del hecho, si presenta la misma información? La labor del entrevistador experto en Psicología y en técnicas de entrevista a través del uso de guías y protocolos debe superar la mera indagación de un hecho, para aportar información experta que oriente de mejor forma la investigación y la decisión del juez. Por lo tanto, el psicólogo o la persona que realiza la entrevista no solo debe estar entrenada en el uso de protocolos, sino que debe poseer un conocimiento sobre el desarrollo a nivel cognitivo, emocional y social de niños, niñas y adolescentes, que le permita adaptar el protocolo a las circunstancias individuales de cada uno de los entrevistados.

\section{Referencias}

American Professional Society on the Abuse of Children (1990). Guidelines for Pshychosocial Evaluation of Suspected Sexual Abuse in Young Children. Task Force on the Pshychosocial Evaluation of Suspected Sexual Abuse in Young Children. Chicago, Il: Author.

American Academy of Child and Adolescent Psychiatry. (1997). Practice parameters for the forensic evaluation of children and adolescents who may have been physically or sexually abused. Journal of the American Academy of Child and Adolescent Psychiatry, 36, 423-442.
American Psychological Association, Committee on Professional Practiceand Standards, a Committee of the Board of Professional Affairs (1995). Twenty-four questions (and answers) about professional practice in the area of child abuse. Professional Psychology: Research and Practice, 26, 377-385.

Anderson, J., Ellefson, J., Lashley, J., Lukas, A., Miller, A., Olinger, S., Russell, A. \& Weigman, J. (2010). The Corner House Forensic Interview Protocol: RATAC®. The Thomas M. Cooley Journal of Practical and Clinical Law, 12(2), 193-331.

Bedoya, L.F. (2008). La prueba en el proceso penal colombiano. Fiscalía General de la Nación Escuela de Estudios e Investigaciones Criminalísticas y Ciencias Forenses. [Versión electrónica].

Berlinerblau, V., Nino, M. \& Viola, S. (2013). Guía Buenas Prácticas para el abordaje de niños/as y adolescentes víctimas o testigos de abuso sexual y otros delitos. Argentina: Unicef.

Bow, J. \& Boxer, P. (2003). Assessing Allegations of Domestic Violence in Child Custody Evaluations. Journal of Interpersonal Violence, 18(12), 1394-1410. DOI: $10.1177 / 0886260503258031$.

Bow, J. N., Quinnell, F. A., Zaroff, M. \& Assemany, A. (2002). Assessment of sexual abuse allegations in child custody cases. Professional Psychology: Research and Practice, 33, 566-575.

Bow, J. N. \& Quinnell, F. A. (2001a). Psychologists' current practices and procedures in child custody evaluations: Five years after American Psychological Association Guidelines. Professional Psychology: Research and Practice, 32, 261-268. 
Bow, J. N. \& Quinnell, F. A. (2001b). Critique of child custody evaluations by the legal profession. Manuscript submitted for publication.

Canton, J. \& Cortés, R. (2008). Guía para la evaluación del Abuso Sexual Infantil. España: Pirámide.

Carens, C., Wilson, C. \& Nelson, D. (1999). Extended forensic evaluation when child abuse is suspected: A model and preliminary data. Child Maltreatment, 4(3), 242-254.

Ceci, S. \& Bruck, M. (1993). Suggestibility of the child witness: a historical review and synthesis. Psychological Bulletin, 113(3), 37.

Ceci, S. \& Bruck, M. (1995). Jeopardy in the courtroom: a scientific analysis of children's testimony. Washington, D.C.: American Psychological Association.

Cederborg, A., Orbach, Y., Sternberg, K. \& Lamb, M. (2000). Investigative interviews of child witnesses in Sweden. Child Abuse $\mathcal{E}$ Neglect, 24, 1355-1362.

Center for Child Protection (1973). Sexual Abuse Evidentary Interiew Protocol. Hospital de San Diego.

Colombia. Corte Constitucional (Sala Penal). Sentencia T-117/2013. Extraída de http://www.corteconstitucional.gov.co/relatoria/2013/T-117-13.htm

Colombia. Corte Suprema de Justicia. Proceso 31950, de 19 de agosto de 2009. Magistrado Ponente Sigifredo Espinoza Pérez. Extraído de: file://C:/ Users/usuario1/Downloads/CSJ\%2031950(19. 08-09).pdf
Collins, R., Lincoln, R. \& Frank, M. G. (2002). The effect of rapport in forensic interviewing. Psychiatry, Psychology $\mathcal{E}$ Law, 9, 69-78.

Coulborn, K. (2005). Anatomical Dolls: Their Use in Assessment of Children Who May Have Been Sexually Abused. Journal of Child Sexual Abuse, 14(1), 8-9.

Curran, T., Schacter, D.L., Johnson, M.K. \& Spinks, R. (2001). Brain potentials reflect behavioral differences in true and false recognition. Journal of Cognitive Neuroscience, 13, 201-216.

Di Lorenzo, M. \& García, I. (2010). La violenciaen la pericia psiquiátrica de niños y adolescentes. Revista de Psiquiatría Uruguaya, 74(1), 67-72.

Echeburúa, E., Amor, P.J. \& Corral, P. (2003). Autoinformes y entrevistas en el ámbito de la Psicología Clínica Forense: limitaciones y nuevas perspectivas. Análisis y Modificación de Conducta, 29, 503 522.

Faller, K. C. (2005). Anatomical dolls: Their use in assessment of children who may have been sexually abused. Journal of Child Sexual Abuse, 14(3), 1-21.

Fisher, R. P. \& Geiselman, R. E. (1992). Memory-enhancing techniques in investigative interviewing: The cognitive interview. Springfield, IL: C.C. Thomas.

Goodman, G. \& Aman, C. (1990). Children's use of anatomically detailed dolls to recount anevent. Child Development, 61, 1859-1871.

Grupo de Trabajo del Gobernador para la Justicia del Menor \& Agencia para la Independencia de la Familia (2003). Protocolo de entrevista forense. 
Recuperado el 02 de mayo de 2014 en http:/www. arpap.org/descargas/AS_05_Protocolo_Michigan_Entrevista_Forense_Abuso_Sexual.pdf

Gudjonsson, G. H. \& Clark, N. K. (1986). Suggestibility in police interrogation: A social psychological model. Social Behaviour, 1, 83-104.

Herman, S. (1997). Practice Parameters for Child Custody Evaluation. Journal of the American Academy of Child $\mathcal{E}$ Adolescent Psychiatry, 36(12), 1784-1787.

Hernández, R., Fernández, C. \& Baptista, P. (2010). Metodología de la Investigación. México: McGraw-Hill.

Instituto Nacional de Medicina Legal y Ciencias Forenses (INMLCF) (2010). Guía para la realización de pericias psiquiátricas y psicológicas forenses en niños, niñas y adolescentes presuntas víctimas de delitos sexuales. Extraído de: https://www. medicinalegal.gov.co/documents/48758/78081/ G4.pdf/bc2dd6af-202b-46ad-81a9-a4ad75210c8d

Juárez, J. R. (2006). El menor como testigo: fundamentos y técnicas. En M. A. Soria Verde, Psicología criminal. Madrid: Pearson.

Lamb, M. E., Orbach, Y., Hershkowitz, I., Esplin, P. W. \& Horowitz, D. (2007). A structured forensic interview protocol improves the quality and informativeness of investigative interviews with children: A review of research using the NICHD Investigative Interview Protocol. Child Abuse $\mathcal{B}$ Neglect, 31, 1201-1231.

Lamb, M., Sternberg, K. \& Esplin, P. (1994). Factors influencing the reliability and validity of statements made by young victims of sexual maltreatment. Journal of Applied Develompental Psychology, 15, 255 280.
Lamb, M. E., Orbach, Y., Sternberg, K. J., Hershkowitz, I. \& Horowitz, D. (2000). Accuracy of investigators' verbatim notes of their forensic interviews with alleged child abuse victims. Law and Human Behavior, 24, 699-708.

Ley 1652 de 2013. Diario Oficial de la República de Colombia 48849 del 12 de julio de 2013. Extraído de http://www.alcaldiabogota.gov.co/sisjur/normas/Norma1.jsp?i=53771

Loftus, E. (1979). Eyewitness testimony. Cambridge, Massachusetts: Harvard U. Press.

Loftus, E. F., Levidow, B. \& Duensing, S. (1992). Who remembers best Individual differences in memory for events that occurred in a science museum. Applied Cognitive Psychology, 6, 93-107.

Montero, A. (1991). Derecho Jurisdiccional. III Proceso Penal. Barcelona: Bosch.

Ortells, M. (1998). Actos de investigación del procedimiento preliminar penal y actos de prueba del juicio. Revista de Derecho Universidad de Concepción, 204, 117-127.

Perona, A., Bottoms, B. \& Sorenson, E. (2006). Research-Based Guidelines for Children Forensic Interviews. En V. Vierth., B. Bottoms \& A. Perona (Eds.), Ending Child Abuse (pp.81-130). NY: Haworth Press.

Poole, D. \& Lamb, M. (1998). Investigative interviews of children: a guide for helping professionals. Washington, D.C.: American Psychological Association. 
Raskin, D. C. \& Esplin, P. W. (1991). Statement Validity Assessment: Interview procedures and content analysis of children's statements of sexual abuse. Behavioral Assessment, 13, 265-291.

Sachsenmaier, S. \& Watson, H. (1998). Do psychological techniques for investigatig child sexual abuse allegations meet the requirements of scientific evidence and the admissibility of expert witness testimony? Comunicación presentada en la reunión anual de la American Academy of Forensic Sciences. San Francisco, California.

Sandoval, C. (1996). La formulación y el diseño de los procesos de investigación social cualitativos. En C. Sandoval, Investigación cualitativa (pp.111-128). Bogotá: Instituto Colombiano para el Fomento de la Educación Superior.

Saywitz, K. \& Snyder, L. (1996). Narrative elaboration: Test of a new procedure for questioning children. Journal of Consulting and Clinical Psychology, 64, 1347-1357.

Saywitz, K., Lyon, T. \& Goodman, G. (2011). Interviewing children. En J.E.B. Myers (Ed.), The APSAC Handbook on Child Maltreatment, 3a. ed. (pp.337-360). Los Angeles: Sage.

Sociedad Internacional para la Prevención del Abuso y la Negligencia Infantil (2011). Comparando las técnicas de entrevista forenses infantiles NICHD y RATAC - ¿Importan las diferencias? La conexión, 20(1).

Strauss, A. (1987). Qualitative analysis for social scientists. Cambridge, UK: Cambridge University Press.
UNICEF (2003). Directrices sobre la justicia para los niños víctimas y testigos de delitos. http://www. unicef.org.co/Ley/AI/13.pdf. ISBN 0-9686330-72

Vallano, J. \& Schreiber Compo, N. (2011). A comfortable witness is a good witness: Rapport-building and susceptibility to misinformation in an investigative mock-crime interview. Applied Cognitive Psychology, 25(6), 960-970.

Walters, S., Holmes, L., Bauer, G. \& Vieth, V. (2003). Finding Words: Half a nation by 2010: Interviewing children and preparing for court. Alexandria, VA: National Center for Prosecution of Child Abuse.

Yuille, J., Hunter, R., Joffe, R. \& Zaparniuk, J. (1993). Interviewing children in sexual abuse cases. En G.S. Goodman \& B.L. Bottoms (Eds.), Child Victims, Child Witnesses: Understanding and Improving Testimony (pp.95-115). New York: The Guilford Press. 